\title{
EL IMPACTO DE LAS ESTRATEGIAS, COLABORACIÓN Y ACUERDOS CON LOS PROVEEDORES: ELEMENTOS CLAVES PARA EL RENDIMIENTO DE LA PYME MANUFACTURERA EN AGUASCALIENTES, MÉXICO
}

\author{
The impact of strategies, and collaboration agreements with suppliers: key elements performance of \\ manufacturing SMEs in Aguascalientes, México
}

\section{Octavio Hernández Castorena}

Doctor en Ciencias Administrativas. Universidad Autónoma de Aguascalientes, Aguascalientes - México, ohernandez@correo.uaa.mx

\section{Luis Aguilera Enríquez}

Doctor en Administración. Universidad Autónoma de Aguascalientes, Aguascalientes - México, laguiler@correo.uaa.mx

\section{Sandra Yesenia Pinzón Castro}

Doctora en Administración. Universidad Autónoma de Aguascalientes, Aguascalientes - México, sypinzon@correo.uaa.mx

\section{Cómo citar / How to cite}

Hernández, O., Aguilera, L. y Pinzón, S. (2015). El impacto de las estrategias, colaboración y acuerdos con los proveedores: elementos claves para el rendimiento de la Pyme manufacturera en Aguascalientes, México. Revista CEA, 1(2), 15-24.

Recibido: 5 de noviembre de 2014

Aceptado: 12 de marzo de 2015

\section{Resumen}

El presente trabajo de investigación muestra los resultados obtenidos a través de la técnica de regresión, con el objetivo de analizar si las estrategias, colaboración y acuerdos que se sostienen con los proveedores tienen impacto significativo para un mayor rendimiento en la Pyme manufacturera en el Estado de Aguascalientes, México. Para ello, se elaboró un instrumento de evaluación dirigido a los gerentes y dueños de este tipo de organizaciones, aplicado de manera transversal, a través de un trabajo empírico en el período de agosto a diciembre del 2013. El estudio indica que para la Pyme manufacturera es importante tener una relación cercana con los proveedores, en virtud de que por la naturaleza de los suministros es necesario integrar estrategias que permitan garantizar las entregas a tiempo y para ello es relevante tener una estrecha colaboración y generar acuerdos que finalmente permita un mayor rendimiento en este tipo de organizaciones. El estudio está enfocado en empresas del sector manufacturero, y el diseño metodológico tiene un enfoque cuantitativo, con alcance descriptivo y correlacional, puesto que para el estudio es necesario analizar la relación entre las variables Estrategias con proveedores, Colaboración y Acuerdos con los proveedores y el Rendimiento.

Palabras clave: estrategias con los proveedores, colaboración y acuerdos con los proveedores, rendimiento, Pyme manufacturera.

\begin{abstract}
This research shows the results obtained through the regression technique in order to analyze whether the strategies, partnerships and agreements with suppliers are having a significant impact for greater performance in manufacturing SMEs in the state Aguascalientes, Mexico. To do this, an assessment tool intended for managers and owners of these organizations applied transversely through an empirical study in the period August to December 2013. The study indicates that developed for the manufacturing SME is important to have a close relationship with suppliers by virtue of the nature of the supplies, it is necessary to integrate strategies to ensure on-time deliveries and it is therefore important to have a close collaboration and generate agreements that eventually allows a higher yield in this types of organizations. The study is focused on companies in the manufacturing sector and the methodological design has a quantitative approach with descriptive and correlational scope since the study is necessary to analyze the relationship between variables Strategies with suppliers, Collaboration and Agreements with suppliers and Performance
\end{abstract}

Keywords: strategies with suppliers, collaboration and agreements with suppliers, performance, SME manufacturing. 


\section{INTRODUCCIÓN}

En la actualidad, la relación entre los proveedores $y$ requisitores se ha sometido a cambios dramáticos, generados en parte por las necesidades naturales de los clientes (Gentry, 1996), es por ello que la relación entre proveedores y compradores ha requerido implementar estrategias enfocadas en la colaboración y en permitir que la gestión de negocios faciliten el intercambio de información que beneficie el desempeño, tanto de los proveedores como de las empresas requisitoras (Ellram, 1990; Hernández, Aguilera \& Colín, 2013; Hernández \& Aguilera, 2014), con la finalidad de que el suministro de los recursos materiales con el apoyo de la implementación de estrategias garantice el suministro en las empresas requisitoras (Lee \& Klaseen, 2008).

En este sentido, las estrategias que requieran integrarse en la relación proveedor-empresa, particularmente en la Pequeña y Mediana Empresa (Pyme) manufacturera, deben enfocarse en tener mayor control en la calidad del manejo de los recursos materiales, reducción en los costos de operación, mejorar el intercambio de información relacionada con el suministro, tener una evaluación de riesgos en la gestión del mismo y volver eficiente la colaboración, así como los acuerdos con la proveeduría en general (Handfield, Sroufe \& Walton, 2005; Burt, 1989; Ellram, 1991; Hendrick y Ellram, 1993; Landeros y Monczka, 1989).

Por otro lado, para las empresas requisitoras es importante que el rendimiento de las actividades operativas en particular con el suministro sea mayor, y para ello, es necesario que la gestión con la proveeduría esté enfocada en mejorar la gestión con los clientes, cuidar los tiempos del suministro y mejorar cualquier actividad operativa que afecte potencialmente la entrega de los recursos materiales (Ross \& Buffa, 2009; Ernst \& Bamford, 2005). En este sentido, el rendimiento puede incrementarse si se integran herramientas tecnológicas que faciliten el manejo de la información sobre el mejor control del suministro de los recursos materiales (Wee, Peng \& Wee, 2010; Kumar, 2001; Walter, Ritter \& Gemunden, 2001; Varma, Wadhva \& Deshmukh, 2006; McLaren, Head \& Yuan, 2002).

El presente estudio tiene como objetivo general profundizar si las estrategias, colaboración y acuerdos que se dan con los proveedores tienen un impacto positivo en el rendimiento de la Pymes manufacturera, ubicadas en Aguascalientes, para que los empresarios o dueños puedan tener información apropiada que pueda incidir para la toma de decisiones en el tema de suministros y de gestión con sus proveedores. Para ello, es importante cuestionar si, por un lado, las estrategias que integren los proveedores podrán ser elementos clave para que se incremente el rendimiento en las Pymes manufactureras; y por otro lado, si la colaboración, así como acuerdos que se tengan con la proveeduría serán elementos esenciales para tener un mayor rendimiento en la eficiencia de los suministros de recursos materiales.

\section{MARCO TEÓRICO}

\section{La relación entre las estrategias con los proveedores y el rendimiento de la Pyme manufacturera}

Para la Pyme manufacturera es de vital importancia que la relación con los proveedores sea en términos de servicio y de buenas prácticas, y para ello, la coordinación que se tenga en el manejo del suministro requiere de estrategias eficientes y correctamente aplicadas (Speakman, 1988; Hernández et al., 2013). Las estrategias que requieran implementarse deben ser acordes con las características particulares de cada organización, sin embargo, por naturaleza, existen aspectos comunes, sobre todo en la Pyme manufacturera, que permiten a los empresarios tomar decisiones relacionadas con las entregas de los suministros para que los resultados en este grupo de organizaciones sean similares (Foster, 1993; Feeney, Lacity \& Wilcox 2005; Handfield \& 
Nichols, 1999; FitzGerald, 1995; Krause, 1997; Adam \& Swamidass, 1989).

Desde luego, para los proveedores es importante tener la capacidad de suministro, puesto que esto permite tener un mayor desarrollo al margen de lo complejo que sea el mercado y la competencia (Lee \& Klassen, 2008). Para ello, es necesario que los proveedores dimensionen lo importante que es implementar estrategias que permitan evitar al máximo las fallas y demoras en el suministro (Hitchens, Trainor, Clausen, Thankapan \& Marchi, 2003). En este sentido, y tal como lo resaltan Bakker \& Nijhof (2002) en su trabajo de investigación, parte de las estrategias están centradas en la habilidad que tienen los proveedores para planear sus actividades internas y de esta manera no tener fallas con el suministros, además de contar con herramientas internas que les permitan autoevaluarse para cumplir al máximo con los compromisos que tengan con sus respectivos clientes.

Asimismo, es importante resaltar que la integración de estrategias debe tener beneficios, tanto para los proveedores como para las Pymes adquisitoras (Chen \& Paulraj, 2004), con el objetivo particular de establecer una relación de negocios entre estos actores a largo plazo (Hahn, Watts \& Kim, 1990; Gadde \& Hakansson, 1994; Swink \& Zsidisin, 2006), para ello, existen algunas estrategias que fortalecen esta relación entre los proveedores y las empresas adquisitoras, las cuales están centradas en la calidad del servicio, la rapidez de respuesta ante cualquier emergencia o el requerimiento de los clientes respecto a alguna necesidad de compra, y la adaptabilidad por parte del proveedor ante cualquier cambio generado por el entorno actual y dinámico de los negocios (Lovello, Saw \& Stimson, 2005).

\section{La relación entre la colaboración y acuerdos con los proveedores y el rendimiento de la Pyme manufacturera}

Desde siempre, la Pyme manufacturera ha tenido un interés muy particular en resolver problemas relacionados con el suministro, sin embargo, en la actualidad, las empresas consideran que el abastecimiento de los recursos materiales requiere de una coordinación especial entre los actores involucrados con los suministros (Van der Vorst, et al., 2002; Lovello, et al., 2005). En este sentido, Wisner (2003) refiere en sus investigaciones la importancia que tiene el control del manejo de los recursos materiales una vez que se tiene una adecuada comunicación con los proveedores, ya que la coordinación, colaboración y acuerdos son esenciales para una buena gestión del suministro $y$, desde luego, con el enfoque de tener un buen rendimiento en las empresas (Hernández \& Aguilera, 2014; Hernández et al., 2013; Kanji \& Wong, 1999).

Para la Pyme manufacturera, la cercanía con los proveedores no solo beneficia la gestión de compra y su rendimiento sino que genera importantes beneficios: optimización de los programas de producción, reducción de costos de operación, mejora en la calidad de servicio y comunicación constante durante la gestión del abastecimiento (Gentry, 1996). Por otro lado, la coordinación con los proveedores requiere profundizar sobre el tipo de productos que maneja en términos de incidir en la mejora de su funcionabilidad, conocer la capacidad de sus procesos internos, evaluar la factibilidad de su organización, su estrategia para establecer relaciones de negocios a largo plazo y conocer sus estrategias de suministro (Rhee \& Lee, 2003; Bowen, Cousins, Lamming \& Faruk, 2001; Tischner, Schmincke, Rubik \& Prosier, 2000; Stafford, Polonsky \& Hartman, 2000; Azzone, Bianchi, Mauri \& Noci, 1997; Zhang, Kuo, Lu \& Huang, 1997).

Es importante resaltar que en la relación con los proveedores, un aspecto importante a desarrollar es el compartir información útil para el abastecimiento de los requerimientos por parte de los requisitores, ya que las empresas, como las Pymes manufactureras, al tener procesos de manufactura requieren una coordinación eficiente entre las necesidades del proceso (en base a las solicitudes de cliente final), los servicios externos como es el caso de intermediarios donde se 
integra el servicio de outsoursing, y la capacidad de suministro por parte del proveedor (Wee et al., 2010; Swink \& Zsidisin, 2006; Fawcett \& Fawcett, 1995; Weber, Current \& Benton, 1991), lo cual conlleva a que este tipo de empresas tengan un mayor rendimiento operativo y un mayor desarrollo al margen de la competencia y de las exigencias actuales del mercado (Hernández \& Aguilera, 2014; Sezhiyan \& Nambirajan, 2010; Chen, Paulraj \& Lado, 2004).

\section{METODOLOGÍA}

El presente trabajo de investigación está enfocado en analizar el impacto que tienen las estrategias, la colaboración y los acuerdos que se tengan con los proveedores, con la finalidad de analizar también si estos elementos son factores claves para que la Pyme manufacturera tenga un mayor rendimiento (Ross \& Buffa, 2009; Wisner, 2003; Sezhiyan \& Nambirajan, 2010; Wee et al., 2010; Gentry, 1996; Lee \& Klassen, 2008), y para ello se plantean las siguientes hipótesis:
$\mathrm{H}_{1}$ : Integrar y aprovechar estrategias con los proveedores permitirá un mayor rendimiento en la Pyme manufacturera de Aguascalientes, México.

$\mathrm{H}_{2}$ : La colaboración y acuerdos con los proveedores, permitirá un mayor rendimiento en la Pyme manufacturera de Aguascalientes, México.

En el presente trabajo se analiza el impacto de las estrategias, colaboración y acuerdos con los proveedores, considerados elementos claves para el rendimiento de la Pyme manufacturera en Aguascalientes, México, en donde se muestra el diseño metodológico de la investigación, el cual por su enfoque se define como cuantitativo. En el presente estudio participó un $76 \%$ de empresas pequeñas y un $24 \%$ de empresas medianas. Como contexto metodológico, el presente estudio es considerado de carácter descriptivo y correlacional (Hernández, Fernández y Baptista, 2006), en la Tabla 1 se integran datos relacionados con el diseño metodológico de la investigación:

Tabla 1. Características del diseño de la investigación

Table 1. Design features of the research

\begin{tabular}{ll}
\hline Indicador de estudio & Descripción \\
\hline Universo & $\begin{array}{l}\text { Empresas pequeñas y medianas del sector manufacturero las } \\
\text { cuales tienen entre } 11 \text { y } 250 \text { empleados }\end{array}$ \\
\hline Ámbito geográfico & Local \\
\hline Tamaño de la población & 442 \\
\hline Tamaño de la muestra & 288 \\
\hline Procedimiento muestral & $\begin{array}{l}\text { Muestreo estratificado proporcional al tamaño y sector de la } \\
\text { empresa }\end{array}$ \\
\hline Error muestral & $+/-8$ \\
\hline Nivel de confianza & $95 \%$ Z $=1.96 ;$ p = q = 0.5 \\
\hline Periodo de realización del & Agosto-diciembre del 2013 \\
trabajo de campo & Fuente: Elaboración propia \\
\hline
\end{tabular}

Se diseñó un instrumento de evaluación dirigido a los gerentes o dueños a través de un trabajo transversal y de trabajo de campo. Para la elaboración del instrumento de medición se integraron los siguientes bloques: el primer bloque se identifica como ESTRATEGIAS CON LOS PROVEEDORES y contiene 11 ítems medidos con escala Likert 1-5, los cuales están 
operacionalizados, desde poca hasta alta importancia (Wisner, 2003), y la escala clasificados en la base de datos como CSO1 a CS11.

El segundo bloque, se identifica como COLABORACIÓN Y ACUERDOS CON LOS PROVEEDORES y contiene 9 ítems medidos con escala Likert 1-5, los cuales están operacionalizados, desde poca hasta alta importancia (Wisner, 2003), y la escala clasificados en la base de datos como CS12 a
CS20. Finalmente, el tercer bloque se identifica como RENDIMIENTO, el cual contiene 12 ítems medidos con escala Likert 1-5 y operacionalizados desde poca hasta alta importancia (Quinn \& Rohrbaugh, 1983), y la escala clasificados en la base de datos como RE01 a RE12.

A continuación, en la Fig. 1 se muestra el modelo teórico diseñado para el presente trabajo de investigación:

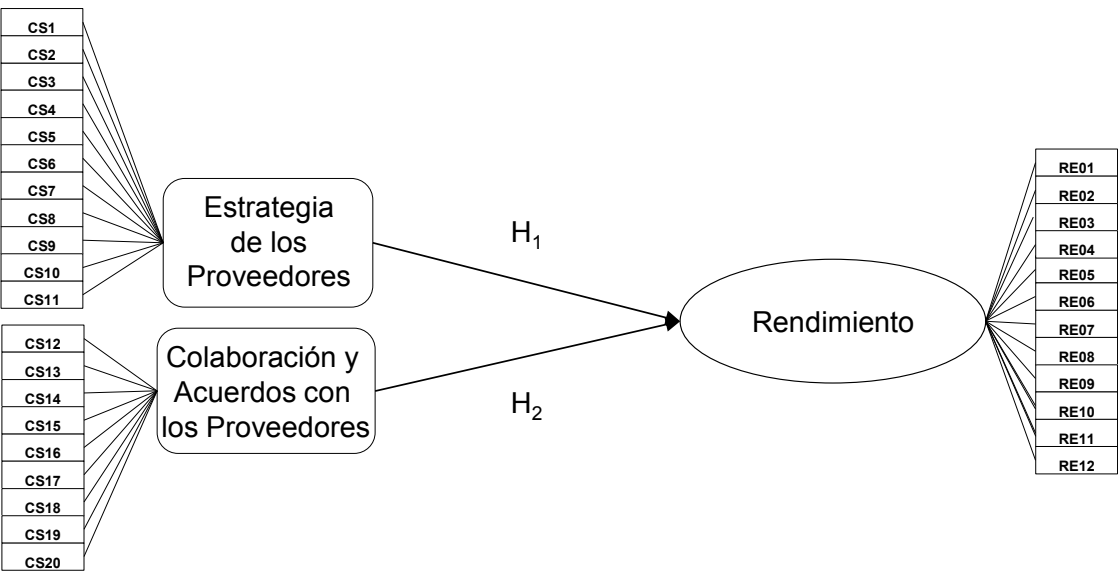

Figura 1. Modelo Teórico de la Investigación

Figure 1. Theoretical Model of Research

Fuente: Elaboración propia a partir de Wisner (2003), Quinn \& Rohrbaugh (1983)

\section{RESULTADOS Y DISCUSIÓN}

Se aplicó un análisis multivariante de datos a través de la técnica estadística de regresión lineal, bajo el método de pasos sucesivos a través del software estadístico IBM SPSS V19, a fin de probar las hipótesis de investigación. A continuación, en la Tabla 2 se presenta el primer resumen del modelo, en el que se obtuvo un valor de $R$ de 0.763 , y una $R^{2}$ de 0.582 , lo que indica que la variable Estrategias con proveedores está correlacionada en un $76.3 \%$ con la variable de Rendimiento en la Pyme manufacturera, y la estructura del modelo teórico se explica en un $58.2 \%$.
Tabla 2. Resumen del primer modelo ${ }^{c}$ Table 2. Summary of the first model ${ }^{c}$

\begin{tabular}{|c|c|}
\hline Modelo & 1 \\
\hline $\mathrm{R}$ & $0.763^{a}$ \\
\hline $\mathrm{R}$ cuadrado & 0.582 \\
\hline R cuadrado corregida & 0.580 \\
\hline $\begin{array}{l}\text { Error típico de la } \\
\text { estimación }\end{array}$ & 0.43067 \\
\hline Durbin-Watson & 1.800 \\
\hline \multicolumn{2}{|c|}{$\begin{array}{l}\text { ESTRATEGIAS CON LOS PROVEEDORES } \\
\text { c. Variable dependiente: RENDIMIENTO }\end{array}$} \\
\hline $\begin{array}{r}\text { Fuente: Elaboración p } \\
\text { datos obtenidos en } \\
\text { versió }\end{array}$ & $\begin{array}{l}\text { artir de los } \\
\text { are SPSS }\end{array}$ \\
\hline
\end{tabular}


Así mismo, se presenta en la Tabla 3, el segundo resumen del modelo referente a la relación de las variables Colaboración y acuerdos con el proveedor con el Rendimiento, en el que se obtuvo un valor de $R$ de 0.671 , y una $R^{2}$ de 0.451 , lo que indica que la colaboración y acuerdos con los proveedores están correlacionadas en un 67.1\% con el Rendimiento de la Pyme manufacturera de Aguascalientes, y el modelo teórico es explicado en un $45.1 \%$.

Tabla 3. Resumen del segundo modelo ${ }^{b}$ Table 3. Summary of the second model ${ }^{b}$

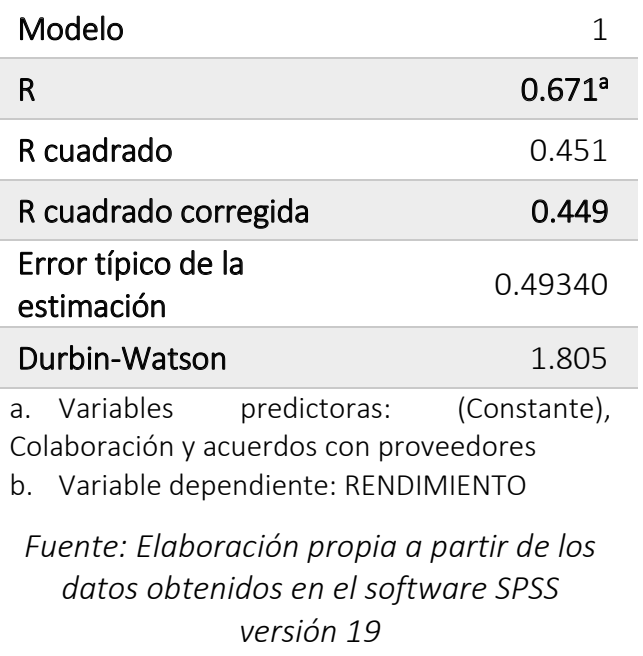

Ahora bien, de acuerdo a los resultados de la regresión lineal que se presentan en la Tabla 4, se concluye que alrededor del $76.3 \%$ del rendimiento de la Pyme manufacturera se debe a las estrategias que tienen los proveedores, al influir esta de manera significativa en el rendimiento, con un valor de t de 9.065, a un nivel de significancia de 0.001; así mismo, la colaboración y acuerdos con el proveedor influyen de manera significativa en un $67.1 \%$ en el rendimiento de la Pyme manufacturera, ya que su valor de $\mathrm{t}$ es de 13.945, a un nivel de significancia de 0.001; y en cuanto a los estadísticos de colinealidad, se obtuvo un factor de inflación de la varianza (FIV) de 1.000, lo que indica que el modelo no presenta problemas de multicolinealidad porque su valor está cercano a la unidad (Hair et al., 1998).
Tabla 4. Resultados Análisis de Regresión Lineal Múltiple

Table 4. Results Multiple Linear Regression Analysis

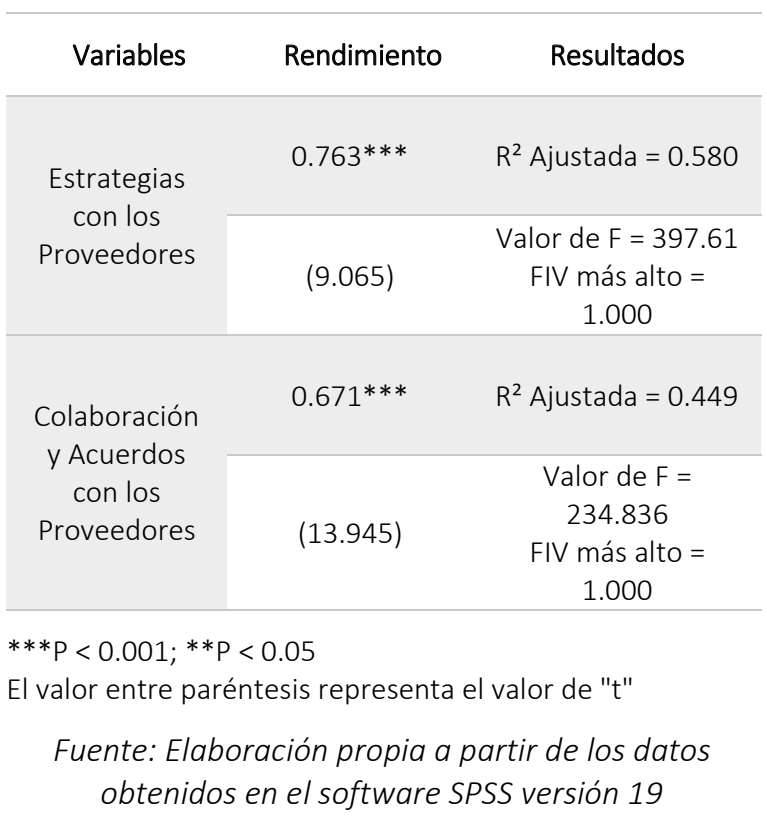

Por lo tanto, en lo que respecta a las hipótesis formuladas en la presente investigación, se concluye lo siguiente: $\mathrm{Con}$ respecto a la $\mathrm{H}_{1}$, los resultados obtenidos $(\beta=0.763, p<0.001)$ indican que las estrategias con proveedores tienen efectos positivos y significativos en el rendimiento de la Pyme manufacturera, por lo tanto al influir en un $76.3 \%$, se acepta la $\mathrm{H}_{1}$. Respecto a la $\mathrm{H}_{2}$, los resultados obtenidos $(\beta=$ $0.671, p<0.001$ ), indican que la colaboración y acuerdos con los proveedores tienen efectos significativos en el rendimiento de la Pyme manufacturera, al influir esta variable en un $67.1 \%$ se acepta la $\mathrm{H}_{2}$.

Finalmente, en lo que respecta a las ecuaciones de regresión, en cuanto al primer modelo se presenta el valor de Y1 que representa el rendimiento de las Pymes manufactureras de Aguascalientes, lo que nos indica que de acuerdo con la ecuación de regresión, el rendimiento está en función de la variable de estrategias con los proveedores en una media de 4.1798, utilizando 1 error estándar. Ver (1) y (2). 


$$
\begin{aligned}
& \mathrm{Y} 1=\beta_{0}+\left(\beta_{1} * \text { Estrategias con los proveedores }\right) \pm e(1) \\
& \text { Rendimiento }=1.325+(0.687 * 4.1798)+/-0.146
\end{aligned}
$$

En cuanto al segundo modelo, se presenta a continuación el valor de Y1 que representa el rendimiento de las Pymes manufactureras de Aguascalientes, lo que nos indica que de acuerdo con la ecuación de regresión, el rendimiento está en función de la variable colaboración y acuerdos con los proveedores en una media de 3.995, utilizando 1 error estándar. Ver (3) y (4).

$$
\begin{gathered}
\mathrm{Y} 1=\beta_{0}+\left(\beta_{1} * \text { Colaboración y acuerdos con los proveedores }\right) \pm e \text { (3) } \\
\text { Rendimiento }=2.021+(0.545 * 3.995) \pm 0.145 \text { (4) }
\end{gathered}
$$

Finalmente, es importante resaltar que el modelo teórico propuesto en el presente estudio refleja la importancia que tiene para la Pyme manufacturera del estado de Aguascalientes en México, al considerar según los resultados obtenidos, que para generar mayores resultados en el rendimiento operativo de la empresa enfocado en la gestión de los suministros es muy importante que las empresas manufactureras tengan una adecuada relación de trabajo con los proveedores, en donde se centren esfuerzos en integrar estrategias con los proveedores, así como tener una colaboración y acuerdos que permitan un suministro libre de riesgo en las entregas a tiempo.

\section{CONCLUSIONES}

De acuerdo con Sezhiyan \& Nambirajan (2010), la selección de los proveedores tiene una influencia relevante en el suministro de los recursos materiales, teniendo con ello un efecto significativo en el rendimiento de las organizaciones. En este sentido, Carr \& Pearson $(1997,1999)$ resaltan la importancia que tiene para las empresas que los proveedores realicen buenas prácticas que influyan en el rendimiento tanto de sus propias empresas como en las de sus clientes. Por otro lado, Ross \& Buffa (2009) mencionan en sus resultados que los proveedores para ser considerados requieren de ofrecer buenos productos y ser confiables en la entrega a tiempo de los suministros. Es por ello, que acorde a estas aportaciones, los resultados obtenidos en el presente estudio confirmen la importancia que tiene para las empresas manufactureras el contar con proveedores confiables.

Continuando con la discusión teórica, para Wee et al. (2010), consideran que el rendimiento en las organizaciones, en gran parte, se da por la integración del servicio de outsoursing, ya que esta figura actúa como un liberador de responsabilidades para la proveeduría. Gentry (1996) por otro lado, refiere que la gestión con los proveedores debe tener claro que el tema del transporte debe cuidar aspectos como la entrega a tiempo, colaboración con cualquier iniciativa que emerja de cada particular necesidad del suministro, de controlar los inventarios y de tener satisfechos a los clientes. En este sentido, los resultados muestran claramente que actualmente las Pymes manufactureras de Aguascalientes mantienen una relación de colaboración con la proveeduría, consintiendo con ello las aportaciones de Wee et al., (2010), y Gentry (1996).

Para Lee \& Klaseen (2008), la capacidad y control de los suministros por parte de los proveedores deben ser eficientes y garantizados, y para ello, como lo muestran los resultados de la presente investigación, es importante que se integren estrategias que mejoren la relación en el tema de suministro por parte de los proveedores para con los clientes, como sucede en la actualidad en 
la Pyme manufacturera de Aguascalientes. Para Wisner (2003), en sus aportaciones resalta la importancia que tiene para una empresa requisitora el tener una colaboración estrecha con los proveedores, en donde es elemental que los acuerdos generados sean sinérgicos y de beneficios para ambas figuras en una relación de negocios, tal y como sucede con las empresas manufactureras en Aguascalientes.

En este sentido, y en base a los resultados obtenidos en el presente estudio sobre lo importante que es la relación entre los proveedores y las empresas pymes manufactureras, se requiere de estrategias que permitan mejorar la relación de negocios para que el rendimiento en este tipo de empresas sea mayor. Por otro lado, los resultados muestran que la colaboración y acuerdos con los proveedores tienen un impacto relevante en el rendimiento de estas empresas, lo cual implica para los dueños o gerentes enfocarse en tener relaciones de negocios que sean a largo plazo, que generen beneficios a sus metas y objetivos empresariales. Sin embargo, los análisis realizados reflejan una clara barrera por parte de los empresarios en compartir información que sea de utilidad a los proveedores para llevar a cabo la gestión del suministro y, en esto, se debe trabajar fuertemente con la finalidad de poder mejorar la relación entre proveedores y clientes.

Finalmente, como limitaciones del presente estudio, es importante resaltar que la muestra se ha desarrollado en el estado de Aguascalientes, México y enfocado en el sector manufacturero, el cual contiene entre 11 y 250 empleados (Pyme). Respecto a las futuras líneas de investigación, se considera importante enfocar el mismo estudio, pero en el tema de competitividad e innovación, sobre todo porque, profundizar sobre la gestión que debe llevarse a cabo con los proveedores requiere, no solamente de colaboración y acuerdos, sino de analizar cuáles estrategias deben implementarse para que el mismo proveedor tenga mayor crecimiento y sea pilar en materia de proveeduría en la región, para con ello evitar al máximo que empresas transnacionales o de gran dinámica de exportación estén pensando en integrar en su cuadro de proveedores a empresas extranjeras.

\section{REFERENCIAS}

Adam, E. E. Jr. \& Swamidass, P. M. (1989). Assessing operations management from a strategic perspective, Journal of Management, 15(2), 181-203.

Azzone, G., Mauri, R. \& Noci, G. (1997). Defining operating enviromental strategies: Programmes and plans within Italian industries, Environmental Management Health, 8(1) 4-19.

Bakker, F. \& Nijhof A. (2012). Responsible chain management: A capability assessment framework, Business Strategy Environmental, 11(1) 63-75.

Bowen, F. E., Cousins, P.D., Lamming, R. C. \& Faruk, A. C. (2001). The role of supply management capabilities in green supply, Production Operations Management, 10(2) 174-189.

Carr, A. S., \& Pearson, J. N. (1997). Strategically managed buyer-supplier relationships and performance outcomes, Decision Sciences Institute, Annual Meeting, Proceedings, 1(3), 1245-1247.

Carr, A. S., \& Pearson, J. N. (1999). Strategically managed buyer-supplier relationships and performance outcomes, Journal of Operations Management, 17(5), 497-519.

Chen, J., Paulraj, A., \& Lado, A. A. (2004). Strategic purchasing, supply management, and firm performance, Journal of Operations Management, 22(5), 505-523.

Chen, I. \& Paulraj, A. (2004). Towards a theory of supply chain management: the constructs and measurements, Journal of Operations Management, 22, 119-150. 
David N.B. (1989). Managing Product Quality Throung Strategic Purchasing, Sloan Management Review, 30(2), 39-48.

Ellram, M. L. (1990). The supplier Selection Decision in Strategic Partnerships, Journal of Purchasing and materials Management, 26(4). 8-14.

Ellram, M. L. (1991). A Managerial Guideline for the Development and Implementation of Purchasing Partnerships, International Journal of Purchasing and Materials Management, 27(3), 2-8.

Ernst, D. \& Bamford, J. (2005). Your alliances are too stable, Harvard Business Review, 83, 133140

Fawcett, S. E., \& Fawcett, S. A. (1995). The firm as a value-added system: integrating logistics, operations and purchasing, International Journal of Physical Distribution \& Logistics Management, 25(5), 24-42.

Feeney, D., Lacity, M. \& Wilcox, L.P. (2005). Taking the measure of outsourcing providers, Sloan Management Review, 46(3), 41-48.

Fitzgerald, K.R. (1995). For Superb Supplier Development-Honda wins! Purchasing 119(4), 32-40.

Foster, A. T. (1993). Brewing Up a Logistics Partnerships, Distribution 92, 49-52

Gadde, L.E \& Hakansson, H. (1994). The changing role of purchasing: reconsidering three strategic issues, European journal of Purchasing and Supply Management, 1, 2735.

Gentry, J.J. (1996). The role of carriers in BuyerSupplier strategic partnerships: A supply Chain management approach, Journal of Business Logistics, 17(2), 35-55.

Hahn, C.K., Watts, C.A. \& Kim, N.Y. (1990). The supply development program: a conceptualmodel. International Journal of Purchasing and Materials Management, 26, 2-7.
Handfield, R., Sroufe, R. \& Walton, S. (2005). Integration environmental management and supply chain strategies. Business Strategy Environmental 14(11) 1-19

Handfield, R. \& Nichols, E.L. (1999). Introduction to Supply Chain management. Upper Saddle River, NJ: Prentice-Hall.

Hernández, O. C. \& Aguilera, E. L. (2014). La gestión de la cadena de suministro: Estrategia clave para un mayor rendimiento en la pyme manufacturera de Aguascalientes. Ideas CONCYTEG 9(107), 2334.

Hernández, O. C., Aguilera, E. L. \& Colín, S. M. (2013). La gestión de los proveedores: Estrategia clave para una mejor gestión de la cadena de suministro en la pyme manufacturera de Aguascalientes. Estudios en Ciencias Sociales y Administrativas de la Universidad de Celaya, 3(1), 9-20.

Hernández, S. R., Fernández, C. C., \& Baptista, L. (2006). Metodología de la investigación. México: McGraw-Hill.

Kanji, G.K. \& Wong, A. (1999). Business excellence model for supply chain management. Total quality management, 10(8), 1147-1168.

Krause, D.R. (1997). Supplier development, current practices and outcomes. International Journal of Purchasing and Materials Management, 33(2), 12-19.

Landeros, R. \& Mencxka, M. R. (1989). Cooperative Buyer/Seller Relationships and a Firm's Competitive Posture. Journal of Purchasing and Materials Management, 25(4), 9-19.

Lee, S.Y. \& Klassen, R.D. (2008). Drivers and Enablers that Foster environmental management capabilities in small and médium sized suppliers in Supply chains. Producton and Operations Management Society, 17(6), 573-586.

Lovello, A., Saw, R. \& Stimson, J. (2005). Product value-density: anaging diversity through 
supply chain segmentation. International Journal of Logistics Management, 16(1), 142-158.

Maclaren, T., Head, M., \& Yuan, Y. (2002). Supply chain collaboration alternative: understanding the expected costs and benefits. Internet Research, 12(4), 348-364.

Rhee, S. \& Lee S. (2003). Dynamic change of corporate environmental strategy: Rhetoric and reality. Business Strategy Environmental, 12(3), 175-190.

Ross, A. \& Buffa, F.P. (2009). Supplier post performance evaluation: the effects of buyer preference weight variance. International Journal of Production Research, 47(16), 4351-4371.

Sezhiyan, D.M \& Nambirajan, T. (2010). An Empirical investigation on relationships between critical suppy Chain management activities and Supplier selection on the business performance Using structural equation model. Journal of International Business and Economics, 10(1), 121-133.

Spekman, E.R. (1988). Strategic Supplier Selection: Understanding Long-Term Buyer Relationships. Business Horizons, 75-81.

Sttaford, E.R., Polonsky, M.J. \& Hartman, C. L. (2000). Environmental NGO-business collaboration and strategic bridging: A case analysis of the Greenpeace-Foron alliance. Business Strategy Environmental, 9(2) 122135.

Swink, M. \& Zsidisin, G. (2006). On the benefits and risks of focused commitment to suppliers. International Journal of Production Research, 44, 4223-4240.

Tischner, U., Schmincke, E., Rubik, F. \& Prosier, M. (2000). How to Do EcoDesing. Verlag form. Frankfurt am Main, Germany.
Thomas E. Hendrick \& Ellram, M. L. (1993). Strategic Supplier Partnering: An International Study, (Temp, Arizona: Center for Advanced Purchasing Studies/National Association of Purchasing Management).

Varma, S., Wadhwa, S., \& Deshmukh, S.G. (2006). Implementing supply chain management in a firm: issues and remedies. Asia Pacific Journal of Marketing and Logistic, 18(3), 22324.

Van der Vorst, J., Beulens, G.A.J., \& Adrie, J.M. (2002). Identifying sources of uncertainty to generate supply chain redesign Strategies. International Journal of Physical Distribution \& Logistics Management, 32(6), 409.

Walter, A., Ritter, T., \& Gemunden, H.G. (2001). Value creation in buyer-seller relationship: theoretical considerations and empirical results from a supplier's perspective. Industrial Marketing Management, 30(4), 365-377.

Weber, C. A., Current, J. R., \& Benton, W. C. (1991). Vendor selection criteria and methods. European Journal of Operational Research, 50(1), 1991, 2-18.

Wee, H.M., Peng, S. Y. \& Wee, P.K.P. (2010). Modelling of outsoursing decisions in global Supply chains, An Empirical Study on Supplier management performance with different outsoursing Strategies., International Journal of Production Reserach, 48(7), 2081-2094.

Wisner, J.D. (2003). A Structural Equation Model of Supply Chain management Strategies and Firm Performance. Journal of Business Logistics, 24(1), 1-26.

Zhang, H. C., Kuo, T. C., Lu, H. \& Huang, S. H. (1997). Environmentally conscious desing and manufacturing: A atate-of-the-art survey. Journal Manufacturing Systems, 16(5) 352-371. 